\title{
T-dual superstring Lagrangian with double zweibeins
}

\author{
Machiko Hatsuda ${ }^{a, b}$ and Warren Siegel ${ }^{c, 1}$ \\ ${ }^{a}$ Department of Radiological Technology, Faculty of Health Science, Juntendo University, \\ Hongo, Bunkyo-ku, Tokyo 113-0033, Japan \\ ${ }^{b}$ KEK Theory Center, High Energy Accelerator Research Organization, \\ Tsukuba, Ibaraki 305-0801, Japan \\ ${ }^{c}$ C.N. Yang Institute for Theoretical Physics, State University of New York, \\ Stony Brook, NY 11794-3840, U.S.A. \\ E-mail: mhatsuda@post.kek.jp, siegel@insti.physics.sunysb.edu
}

ABSTRACT: We present superstring Lagrangians with manifest T-duality. The Lagrangian version of the section conditions are necessary to make Lagrangians to be general coordinate invariant. We show the general solution of section conditions. The D-dimensional left and right moving currents are the 2D-dimensional chiral current which causes the chiral boson problem. We solve the problem by adding the unphysical 2D-dimensional antiselfdual current with the selfduality constraints. The Lagrange multipliers of the selfduality constraints play the role of the worldsheet zweibein allowing the Weyl invariant and Lorentz symmetric worldsheet. Doubling the zweibein makes the type II $\kappa$-symmetry splitting into two sets of the type I $\kappa$-symmetries.

KEYwords: String Duality, Superstrings and Heterotic Strings, Space-Time Symmetries, Supersymmetry and Duality

ARXIV EPRINT: 1912.05092

\footnotetext{
${ }^{1}$ http://insti.physics.sunysb.edu/ siegel/plan.html.
} 


\section{Contents}

1 Introduction 1

2 Section conditions $\quad 3$

2.1 Section conditions in Hamiltonian 3

2.2 Section conditions in Lagrangian 5

$\begin{array}{llr}3 & \text { Worldsheet gauges } & 6\end{array}$

4 Non-abelian space currents $\quad 9$

4.1 Algebra and currents 9

$\begin{array}{lll}4.2 & \text { Virasoro and selfduality constraints } & 10\end{array}$

$5 \quad$ T-dual superstring Lagrangians $\quad 12$

$\begin{array}{lll}5.1 \text { Superalgebras and currents } & 12\end{array}$

$\begin{array}{lll}5.2 & \text { Superstring Lagrangians } & 14\end{array}$

$\begin{array}{llr}6 & \text { Conclusions } & 18\end{array}$

$\begin{array}{lr}\text { A Indices } & 18\end{array}$

\section{Introduction}

T-duality is the characteristic feature of the string theory which leads to a different picture from the Einstein gravity drastically at the short distance. T-duality of string action is made manifest by doubling the spacetime coordinates [1, 2]. Since physical string currents are the D-dimensional left and right currents, the physical current in the doubled spacetime is the 2D-dimensional chiral current which gives rise to the chiral boson problem [3]. A Lagrangian only with chiral currents does not allow the conformal gauge which is useful for the quantum computation. The Weyl invariance and the Lorentz covariance of the worldsheet are necessary especially for a superstring since the $\kappa$-symmetry involves the gauge transformation of the zweibein. Pasti, Sorokin and Tonin introduced a scalar to resolve the chiral scalar problem $[4,5]$ which has a nonzero vacuum value leading to the spontaneous breaking down of the worldsheet symmetry. It was applied to make manifest T-duality in [6].

Bandos proposed the superstring Lagrangian with manifestly T-duality where the PST scalar field is used resulting double zweibeins [7]. The obtained superstring action has two sets of $\kappa$-symmetries leading to simpler structure of the gauge invariance. In our previous paper [8] doubling of the zweibein comes from the Lagrange multipliers of the selfduality constraints for a bosonic string. In this paper we extend it to the supersymmetric case. Our Lagrangian is similar to the one obtained by Bandos [7]. Differences of our 
treatment in this paper from [7] are the followings: (1) We use both the selfdual and anti-selfdual currents which are extendable to non-abelian cases instead of the Hodge dual of the selfdual current in [7]. (2) Lagrange multipliers of the selfduality constraints are used for double zweibeins, instead of the PST scalar. (3) The Wess-Zumino terms are written in bilinears of the currents as well as the kinetic term in our approach. We also mention about our "chiral" treatment in the previous paper [9]. The main differrence from [7] is the dimensional reduction constraint which is linear combination of the antiselfdual currents. Although we began by only the selfdual currents [10,21], the dimensional reduction constraints involve the anti-selfdual currents leading to the Weyl invariant and Lorentz covariant worldsheet Lagrangian. As earlier studies many aspects of superstring Lagrangians with T-duality are examined such as the NS/NS superstring [11], the doubledyet-gauged spacetime formulation [12-14] and the pure spinor [15].

The string background is described by the gravity with the T-duality symmetry. It was shown that the classical gravity theory with manifest T-duality is the same with the low energy 1-loop effective theory of the string [16-18] and the one for the chiral string in the $\alpha^{\prime}$ order [19]. It is the gauge theory of gravity. For the Einstein gravity the gauge generator and the gauge field are the momentum $p_{\mathrm{M}}$ and the vielbein $e_{\mathrm{A}}{ }^{\mathrm{M}}$ which make the covariant derivative $\nabla_{\mathrm{A}}=e_{\mathrm{A}}{ }^{\mathrm{M}} p_{\mathrm{M}}$. For the stringy gravity the momentum includes the winding mode $\triangleright_{M}(\sigma)=\left(p_{\mathrm{M}}, \partial_{\sigma} x^{\mathrm{M}}\right)$ with the D-dimensional index $\mathrm{M}$ and the $2 \mathrm{D}$-dimensional index $M$. They satisfy the affine Lie algebra whose consistency requires the nondegenerate group metric $\eta_{M N}$. This affine Lie algebra is realized by the $2 \mathrm{D}$-dimensional canonical coordinates $P_{M}$ and $X^{M}$. The covariant derivative is extended to $\nabla_{\mathrm{A}} \rightarrow \triangleright_{A}(\sigma)=E_{A}{ }^{M} \triangleright_{M}$ with the vielbein $E_{A}{ }^{M}(X)$. The gauge transformation of the vielbein under the general coordinate transformation is given by $\delta E_{A}{ }^{M}=d E_{A}{ }^{M}+\mathcal{L}_{\Lambda} E_{A}{ }^{M}$ with the differential term $d E_{A}{ }^{M}$ and the "new" Lie derivative $\mathcal{L}_{\Lambda} E_{A}{ }^{M}$. The "new" Lie derivative gives rise to the $\mathrm{O}(\mathrm{D}, \mathrm{D})$ transformation

$$
\delta E_{A}{ }^{M}=E_{A}^{N}\left(-\partial_{N} \Lambda^{M}+\partial^{M} \Lambda_{N}\right), \quad \delta E_{M}^{A}=\left(\partial_{M} \Lambda^{N}-\partial^{N} \Lambda_{M}\right) E_{N}{ }^{A} .
$$

Throughout this paper the 2D-dimensional index $M$ is raised and lowered by the $\mathrm{O}(\mathrm{D}, \mathrm{D})$ invariant metric $\eta^{M N}$. The tangent vector is transformed as $\mathrm{O}(\mathrm{D}, \mathrm{D})$

$$
\frac{\partial}{\partial X^{\prime M}}=\frac{\partial X^{N}}{\partial X^{\prime M}} \frac{\partial}{\partial X^{N}}=\left(\delta_{M}^{N}+\partial_{M} \Lambda^{N}\right) \partial_{N} \approx\left(\delta_{M}^{N}+\partial_{M} \Lambda^{N}-\partial^{N} \Lambda_{M}\right) \partial_{N}
$$

where the section conditions on arbitrary functions $\Psi_{I}(X)$ with $I=1,2, \cdots$ are used

$$
\eta^{M N} \partial_{M} \partial_{N} \Psi_{I}(X)=\eta^{M N} \partial_{M} \Psi_{I}(X) \partial_{N} \Psi_{J}(X)=0
$$

The cotangent vector should be also transformed as $\mathrm{O}(\mathrm{D}, \mathrm{D})$

$$
d X^{\prime M}=d X^{N} \frac{\partial X^{\prime M}}{\partial X^{N}}=d X^{N}\left(\delta_{N}^{M}-\partial_{N} \Lambda^{M}\right) \approx d X^{N}\left(\delta_{N}^{M}-\partial_{N} \Lambda^{M}+\partial^{M} \Lambda_{N}\right),
$$

so the Lagrangian version of the section conditions are necessary as shown in our previous paper [8]

$$
d X^{M} \eta_{M N} d X^{N}=d X^{\prime M} \eta_{M N} d X^{\prime N}=0=d X^{M} \eta_{M N} \partial_{L} \Lambda^{N}
$$


These conditions guarantee the consistency for the cotangent vector $d X^{M}$ and the tangent vector $\frac{\partial}{\partial X^{N}}$ as $\left\langle d X^{M}, \frac{\partial}{\partial X^{N}}\right\rangle=\delta_{N}^{M}$. The Lagrangian version of the section conditions also guarantee the coordinate invariance of the currents in curved backgrounds

$$
J_{m}{ }^{A}=\partial_{m} X^{M} E_{M}{ }^{A}, \delta J_{m}{ }^{A}=\left(\partial_{m} \delta X^{M}\right) E_{M}{ }^{A}+\partial_{m} X^{M} \delta E_{M}{ }^{A}=0,
$$

where index $m$ runs $\tau, \sigma$ for a string and $\tau$ for a particle. Then the Lagrangian is made to be coordinate invariant.

The organization of the paper is the following: in the next section we present solutions of the section conditions in Hamiltonian (1.3) and in Lagrangian (1.5) explicitly. In section 3 , the worldsheet gauges are examined where the Lagrange multipliers of the Virasoro constraints and the selfduality constraints in Hamiltonian become double zweibeins. In section 4 the Virasoro constraints and the selfduality constraints in a non-abelian space are obtained. We begin by the Hamiltonian formalism where the covariant derivative commutes with the symmetry generator. The covariant derivative is the selfdual current while the orthogonal transformed symmetry generator becomes the anti-selfdual current. The $\sigma$ diffeomorphism Virasoro operator includes the anti-selfdual current, so the $\sigma$ derivative computed by the canonical commutator coincides with the one computed by the chain rule differential. Including the anti-selfdual current is a similar formulation given in [20] where the Lagrangian for selfdual 2n-form fields is written with the anti-seldfual form.

In section 5, the superstring Lagrangians with manifest T-duality are presented. For two sets of nondegenerate superalgebras the selfdual and anti-selfdual currents are given concretely. The Hamiltonian of the superstring includes the Virasoro constraints, the selfduality constraints and the dimensional reduction constraints for unphysical fermions. The Lagrangian with double zweibeins makes the type II $\kappa$-symmetry to be two sets of the type I $\kappa$-symmetries leading to simpler computation. We also show how to reduce to the Green-Schwarz superstring action by gauge fixing and sectioning.

\section{Section conditions}

\subsection{Section conditions in Hamiltonian}

The manifestly T-duality space is defined by the string current algebra, where the worldsheet spatial diffeomorphism is suppressed consistently as the section conditions. The Virasoro operators are

$$
\left\{\begin{array}{l}
\mathcal{H}_{\tau}=\frac{1}{2} P_{M} G^{M N} P_{N} \\
\mathcal{H}_{\sigma}=\frac{1}{2} P_{M} \eta^{M N} P_{N}
\end{array}\right.
$$

where $G^{M N}$ is the $\mathrm{O}(\mathrm{D}, \mathrm{D})$ gravitational background metric while $\eta^{M N}$ is the $\mathrm{O}(\mathrm{D}, \mathrm{D})$ invariant metric. $\mathcal{H}_{\tau}$ is the Hamiltonian in the conformal gauge. $\mathcal{H}_{\sigma}=0$ is realized on arbitrary fields $\Psi_{i}\left(X^{M}\right), i=1,2, \cdots$ as weak and strong section conditions

$$
\eta^{M N} \partial_{M} \partial_{N} \Psi_{i}(X)=\eta^{M N} \partial_{M} \Psi_{i}(X) \partial_{N} \Psi_{j}(X)=0 \text { for } i, j=1,2, \cdots
$$


The Fourier transformation introduces momenta $P_{i ; M}$ for each function as

$$
\Psi_{i}\left(X^{M}\right)=\int d^{2 D} P_{i} e^{-i P_{i ; M} X^{M}} \tilde{\Psi}_{i}\left(P_{i ; M}\right) .
$$

The weak section condition gives

$$
\eta^{M N} \partial_{M} \partial_{N} \Psi_{i}(X)=0 \rightarrow \eta^{M N} P_{i M} P_{i ; N}=0 \text { for } i=1,2, \cdots .
$$

Let us divide the 2D directions of $P_{M}$ into two Euclidean D-dimensional directions of the positive metric $\bar{P}_{\bar{M}}$ and the one of the negative metric $\underline{P}_{M}$

$$
\eta^{M N} P_{i ; M} P_{i ; N}=\sum_{\bar{M}=1}^{D}\left(\bar{P}_{i ; \bar{M}}\right)^{2}-\sum_{\underline{M}=1}^{D}\left(\underline{P}_{i ; \underline{M}}\right)^{2}=0 \rightarrow \sum_{\bar{M}=1}^{D}\left(\bar{P}_{i ; \bar{M}}\right)^{2}=\sum_{\underline{M}=1}^{D}\left(\underline{P}_{i ; \underline{M}}\right)^{2} \equiv\left|P_{i}\right|^{2}
$$

The strong section condition gives

$$
\eta^{M N} \partial_{M} \Psi_{i}(X) \partial_{N} \Psi_{j}(X)=0 \rightarrow \eta^{M N} P_{i ; M} P_{j ; N}=0 \text { for } i \neq j=1,2, \cdots
$$

which leads to

$$
\begin{aligned}
\eta^{M N} P_{i ; M} P_{j ; N} & =\sum_{\bar{M}=1}^{D} \bar{P}_{i ; \bar{M}} \bar{P}_{j ; \bar{M}}-\sum_{\underline{M}=1}^{D} \underline{P}_{i ; \underline{M}} \underline{\underline{P}} \underline{I}_{j ; \underline{M}}=\left|P_{i}\right|\left|P_{j}\right|\left(\cos \bar{\theta}_{i j}-\cos \underline{\theta}_{i j}\right)=0 \\
\Rightarrow \bar{\theta}_{i j} & =\underline{\theta}_{i j} .
\end{aligned}
$$

In order to coincide all angles of infinite number of arbitrary vectors, $\bar{\theta}_{i j}=\underline{\theta}_{i j}$, the positive and negative vectors $\bar{P}_{i ; \bar{M}}$ and $\underline{P}_{i ; \underline{M}}$ must be equal up to an $\mathrm{O}(\mathrm{D})$ rotation $A_{\bar{M}^{\underline{N}}}$

$$
\bar{P}_{i ; \bar{M}}=A_{\bar{M}} \underline{N}_{i ; \underline{N}} \text { for } i=1,2, \cdots, A_{\bar{M}} A_{\bar{M}} \frac{L}{N}=\delta \underline{N L}, A_{\bar{M}} A_{\frac{N}{L}}=\delta_{\overline{M L}} .
$$

The infinite number of vectors $\bar{P}_{i ; \bar{M}}$ and $\underline{P}_{i ; \underline{M}}$ are recognized as infinite number of points in the momentum coordinate space $\bar{P}_{\bar{M}}$ and $\underline{P}_{M}$. Therefore the reducibility (2.7) eliminates a half space as

$$
\bar{P}_{\bar{M}}=A_{\bar{M}} \underline{N}_{\underline{N}} \rightarrow 0=\left(\begin{array}{c|c}
\mathbf{1}_{M^{\underline{N}}} & -A_{\bar{M}^{\underline{N}}} \\
\hline 0 & 0
\end{array}\right)\left(\begin{array}{c}
\bar{P}_{\bar{N}} \\
\hline \underline{P}_{\underline{N}}
\end{array}\right)
$$

Interchanging $\bar{P}_{\bar{D}}$ and $\underline{P}_{\underline{D}}$ of the positive and negative vectors and renaming them as $P_{\underline{0}}$ and $P_{\overline{0}}$ make the Lorentz covariant left and right vectors. The interchanging matrix $\mathcal{I}$ makes the left/right vectors $P_{\mathrm{L} / \mathrm{R}}=\left(P_{\bar{M}}, P_{\underline{M}}\right)$ from the positive/negative vectors $P_{\mathrm{P} / \mathrm{N}}=$ $\left(\bar{P}_{\bar{M}}, \underline{P}_{\underline{M}}\right)$. It also makes the $\mathrm{O}(\mathrm{D}, \mathrm{D})$ invariant metric to be diagonal $\left(\eta_{\overline{M N}},-\eta_{\overline{M N}}\right)$ from diagonal $(\mathbf{1}, \mathbf{- 1})$

$$
\mathcal{I}=\left(\begin{array}{lll|l}
\mathbf{1} & \mathbf{0} & \mathbf{0} & \mathbf{0} \\
\mathbf{0} & 0 & \mathbf{0} & 1 \\
\hline \mathbf{0} & \mathbf{0} & \mathbf{1} & \mathbf{0} \\
\mathbf{0} & 1 & \mathbf{0} & 0
\end{array}\right), P_{\mathrm{L} / \mathrm{R}}=\mathcal{I} P_{\mathrm{P} / \mathrm{N}}, \eta_{M N}=\mathcal{I}\left(\begin{array}{c|c}
\mathbf{1} & \mathbf{0} \\
\hline \mathbf{0} & -\mathbf{1}
\end{array}\right) \mathcal{I}=\left(\begin{array}{c|c}
\eta_{\overline{M N}} & 0 \\
\hline 0 & -\eta_{\overline{M N}}
\end{array}\right)
$$


Plugging (2.9) into the reducibility relation (2.8) the left/right momenta also satisfy the reducibility condition with a new reducibility matrix denoted by the same matrix $A$ as

$$
P_{\bar{M}}=A_{\bar{M}}{ }^{N} P_{\underline{N}}
$$

The matrix $A_{\bar{M}^{N}}$ is an element of $\mathrm{O}(\mathrm{D}-1,1)$ group with respect to $\eta_{\overline{M N}}$ and $\eta^{\overline{M N}}$ as $A \eta A^{T}=\eta$ and $A^{T} \eta A=\eta$. By using $A_{\bar{M}}^{\underline{N}}$ we fix the left and right Lorentz symmetries, $S_{\overline{M N}}$ and $S_{\underline{M N}}$, as

$$
S_{\text {physical;MN }}=S_{\overline{M N}}-A_{\bar{M}} A_{\bar{N}} \frac{K}{\underline{L K}}, \quad 0=S_{\overline{M N}}+A_{\bar{M}} A_{\bar{N}} \frac{K}{\underline{L K}}
$$

It is denoted that the left and the right Lorentz generators satisfy the same Lorentz algebras with the opposite signatures as $\left[S_{\text {left }}, S_{\text {left }}\right]=S_{\text {left }}$ and $\left[S_{\text {right }}, S_{\text {right }}\right]=-S_{\text {right }}$. Linear combination of the left/right momenta brings to the conventional basis $p_{\mathrm{M}}$ and $p^{\mathrm{M}}$ as

$$
\left\{\begin{aligned}
p_{\text {physical; } \mathrm{M}} & =\frac{1}{2}\left(P_{\bar{M}}+A_{\bar{M}} \underline{\underline{L}} P_{\underline{L}}\right)=P_{\bar{M}} \\
p^{\mathrm{M}} & =\frac{1}{2}\left(P_{\bar{M}}-A_{\bar{M}} \underline{\underline{L}} P_{\underline{L}}\right)=0
\end{aligned}\right.
$$

The Fourier functions have the form of $\tilde{\Psi}_{i}\left(P_{i ; M}\right)=\tilde{\Psi}_{i}\left(p_{i ; \mathrm{M}}, p_{i}{ }^{\mathrm{M}}=0\right)$. The Fourier integration with respect to $p_{i}{ }^{\mathrm{M}}$ gives $\int d^{D} p_{i}{ }^{\mathrm{M}} e^{-i p_{i}{ }^{\mathrm{M}} y_{\mathrm{M}}} \tilde{\Psi}_{i}\left(P_{M}\right)=\delta\left(y_{\mathrm{M}}\right) \tilde{\Psi}_{i}\left(p_{\mathrm{M}}\right)$, so the resultant functions are

$$
\Psi_{i}\left(X^{M}\right)=\Psi_{i}\left(x^{\mathrm{M}}, y_{\mathrm{M}}=0\right) .
$$

The half of $2 \mathrm{D}$ coordinates are suppressed. Other solutions such as $x^{\mathrm{M}}=0, y_{\mathrm{M}} \neq 0$, are obtained by regular $\mathrm{O}(\mathrm{D}, \mathrm{D})$ matrix transformations.

\subsection{Section conditions in Lagrangian}

The Lagrangian version of the section conditions are given in our previous paper [8]. We also showed that the Virasoro operators (2.1) become the line element and the constraint in Lagrangian formalism

$$
\left\{\begin{array}{l}
\frac{1}{2} d X^{M} G_{M N} d X^{N}=d^{2} s \\
\frac{1}{2} d X^{M} \eta_{M N} d X^{N}=0
\end{array}\right.
$$

The second line is the section condition suppressing the degrees of freedom generated by the $\sigma$-diffeomorphism which corresponds to the weak section condition. Under an infinitesimal coordinate transformation $X^{M} \rightarrow X^{M}=X^{M}-\Lambda^{M}(X)$ the additional condition is required [8] corresponding to the strong section condition

$$
d X^{M} \eta_{M N} \partial_{L} \Lambda^{N}=0
$$

The Lagrangian version of the section conditions are solved analogously in (2.10)

$$
\eta_{M N} d X^{M} d X^{N}=\eta_{M N} d X^{\prime M} d X^{\prime N}=0, \eta_{M N} d X^{M} d X^{\prime N}=0 \rightarrow d X^{\bar{M}}=d X^{\underline{N}}\left(A^{-1}\right)_{\underline{N}^{M}}
$$


The left and right Lorentz symmetries are fixed analogously to (2.11). The conventional coordinates are introduced as

$$
\left\{\begin{array}{l}
x^{\mathrm{M}}=X^{\bar{M}}+X^{\underline{N}}\left(A^{-1}\right)_{\underline{N}}^{\bar{M}} \\
y_{\mathrm{M}}=X^{\bar{M}}-X^{\underline{N}}\left(A^{-1}\right)_{\underline{\underline{N}}}^{\bar{M}}
\end{array}\right.
$$

A solution of the Lagrangian version of the weak section condition (2.16) is given by

$$
\left\{\begin{array}{l}
d x^{\mathrm{M}}=d X^{\bar{M}}+d X^{\underline{N}}\left(A^{-1}\right)_{\underline{\underline{N}}}{ }^{\bar{M}}=2 d X^{\bar{M}} \\
d y_{\mathrm{M}}=d X^{\bar{M}}-d X^{\underline{N}}\left(A^{-1}\right)_{\underline{\underline{N}}}^{\bar{M}}=0
\end{array} \quad \rightarrow d X^{M}=\left(d x^{\mathrm{M}}, d y_{\mathrm{M}}=0\right) .\right.
$$

This solution supplements the solution of $y_{\mathrm{M}}=0$ in (2.13). The weak condition in (2.15) is examined as $d X^{M} \eta_{M N} \partial_{L} \Lambda^{N}=0=d x^{\mathrm{M}} \frac{\partial}{\partial x^{L}} \Lambda_{\mathrm{M}}=0$ leading to $\Lambda_{\mathrm{M}}=$ constant, where $\frac{\partial}{\partial y_{M}} \Lambda=0$ and $d y_{M}=0$ are used. So the transformed coordinate is still solution $d y_{M}^{\prime}=$ $d y_{\mathrm{M}}-d \Lambda_{\mathrm{M}}=0$. Other solutions are obtained by regular $\mathrm{O}(\mathrm{D}, \mathrm{D})$ matrix transfomations.

\section{$3 \quad$ Worldsheet gauges}

The Lagrange multipliers of the Virasoro constraints in Hamiltonian are zweiben gauge fields in Lagrangian. The choice of the zweibein gauge links the target space symmetry. In order to focus on this relation we consider a bosonic string in a D-dimensional flat space without the $B$-field. The D-dimensional target space coordinate and the canonical conjugate are denoted by $\left(x^{\mathrm{M}}, p_{\mathrm{M}}\right)$ with the usual Lorentz metric $\eta_{\mathrm{MN}}$. The Hamiltonian for a string in the D-dimensional flat space gives the Lagrangian in the Weyl-Lorentz gauge of the zweibein

$$
\begin{aligned}
& H_{0}=g_{-} \frac{1}{4}\left(p+\partial_{\sigma} x\right)_{\mathrm{M}}^{2}+g_{+} \frac{1}{4}\left(p-\partial_{\sigma} x\right)_{\mathrm{M}}^{2} \\
& L_{0}=\partial_{\tau} x^{\mathrm{M}} p_{\mathrm{M}}-H_{0}=\frac{1}{e}\left(e_{+}{ }^{m} \partial_{m} x^{\mathrm{M}}\right)\left(e_{-}{ }^{n} \partial_{n} x^{\mathrm{N}}\right) \eta_{\mathrm{MN}}
\end{aligned}
$$

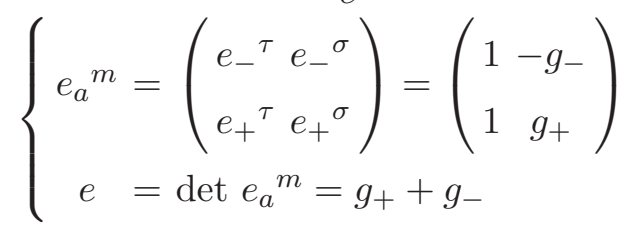

In the manifestly T-duality formulation the D-dimensional left/right coordinates are treated as independent 2D-dimensional coordinates; the coordinates $X^{M}=\left(X^{\bar{M}}, X^{\underline{M}}\right)$ and the conjugate momenta $P_{M}=\left(P_{\bar{M}}, P_{\underline{M}}\right)$. On the other hand the $2 \mathrm{D}$-dimensional left/right moving currents, $P_{M} \pm \partial_{\sigma} X^{N} \eta_{N M}$, include not only the D-dimensional left/right moving currents $P_{M}+\partial_{\sigma} X^{N} \eta_{N M}=\left(\left(P+\partial_{\sigma} X\right)_{\bar{M}},\left(P-\partial_{\sigma} X\right)_{\underline{M}}\right)$ but also unphysical currents $P_{M}-\partial_{\sigma} X^{N} \eta_{N M}=\left(\left(P-\partial_{\sigma} X\right)_{\bar{M}},\left(P+\partial_{\sigma} X\right)_{\underline{M}}\right) . P_{M}+\partial_{\sigma} X^{N} \eta_{N M}$ is the selfdual current and $P_{M}-\partial_{\sigma} X^{N} \eta_{N M}$ is the anti-selfdual currents.

We review our chiral approach [9]. The Hamiltonian includes only physical currents. The selfduality constraint is imposed by the linear combination of the left/right anti-selfdual 
currents in such a way that the stringy anomaly is cancelled, $\left(P-\partial_{\sigma} X\right)_{\bar{M}}-\left(P+\partial_{\sigma} X\right)_{\underline{M}}=0$. The chiral Hamiltonian with the linear selfduality constraint is given by

$$
\begin{aligned}
H_{\text {chiral }}= & g_{-} \frac{1}{4}\left(P+\partial_{\sigma} X\right)_{\bar{M}^{2}}+g_{+} \frac{1}{4}\left(P-\partial_{\sigma} X\right)_{\underline{\underline{M}}}^{2} \\
& +\mu^{\mathrm{M}}\left\{\left(P-\partial_{\sigma} X\right)_{\bar{M}}-\left(P+\partial_{\sigma} X\right)_{\underline{M}}\right\} \\
L_{\text {chiral }}= & \partial_{\tau} X^{M} P_{M}-H_{\text {chiral }} \\
= & \frac{1}{g_{-}}\left\{\left(e_{-}^{m} \partial_{m} X^{\bar{M}}-\mu^{\mathrm{M}}\right)^{2}+g_{-} \partial_{\sigma} X^{\bar{M}}\left(e_{-}{ }^{m} \partial_{m} X^{\bar{M}}\right)\right\} \\
& +\frac{1}{g_{+}}\left\{\left(e_{+}{ }^{m} \partial_{m} X^{\underline{M}}+\mu^{\mathrm{M}}\right)^{2}-g_{+} \partial_{\sigma} X^{\underline{M}}\left(e_{+}{ }^{m} \partial_{m} X^{\underline{M}}\right)\right\}
\end{aligned}
$$

where the zweibein $e_{a}{ }^{m}$ is given in (3.1). After integrating out the Lagrangian multiplier $\mu^{\mathrm{M}}$ and rewriting in terms of the usual coordinates $x^{\mathrm{M}}$ and $y_{\mathrm{M}}$ in (2.17), the worldsheet covariant Lagrangian is obtained

$$
L_{\text {chiral }}=\frac{1}{e}\left(e_{+}{ }^{m} \partial_{m} x^{\mathrm{M}}\right)\left(e_{-}{ }^{n} \partial_{n} x^{\mathrm{N}}\right) \eta_{\mathrm{MN}}-\epsilon^{m n} \partial_{m} x^{\mathrm{M}} \partial_{n} y_{\mathrm{M}} .
$$

The first term is both D-dimensional and worldsheet covariant kinetic term. The second term is total derivative in the bosonic case, but it contributes to the supersymmetric case.

Next let us include the anti-selfdual currents. The Hamiltonian for a string in the 2Ddimensional flat space gives the Lagrangian in the Weyl-Lorentz gauge with two zweibeins:

$$
\begin{aligned}
& H_{1}=g_{-} \frac{1}{4}\left(P+\partial_{\sigma} X\right)_{\bar{M}^{2}}{ }^{2}+g_{+} \frac{1}{4}\left(P-\partial_{\sigma} X\right)_{\underline{M}^{2}} \\
& +\left(g_{+}+\lambda_{+}\right) \frac{1}{4}\left(P-\partial_{\sigma} X\right)_{\bar{M}^{2}}{ }^{2}+\left(g_{-}+\lambda_{-}\right) \frac{1}{4}\left(P+\partial_{\sigma} X\right)_{\underline{M}^{2}} \\
& L_{1}=\partial_{\tau} X^{M} P_{M}-H_{1} \\
& =\frac{1}{\bar{e}}\left(\bar{e}_{+}{ }^{m} \partial_{m} X^{\bar{M}}\right)\left(\bar{e}_{-}{ }^{n} \partial_{n} X^{\bar{N}}\right) \eta_{\overline{M N}}+\frac{1}{\underline{e}}\left(\underline{e}_{+}{ }^{m} \partial_{m} X^{\underline{M}}\right)\left(\underline{e}_{-}{ }^{n} \partial_{n} X^{\underline{N}}\right) \eta_{\overline{M N}} \\
& \left\{\begin{array}{c}
\bar{e}_{a}^{m}=\left(\begin{array}{cc}
1 & -g_{-} \\
1 & g_{+}+\lambda_{+}
\end{array}\right) \\
\bar{e}=e+\lambda_{+}
\end{array},\left\{\begin{array}{c}
\underline{e}_{a}^{m}=\left(\begin{array}{cc}
1-\left(g_{-}+\lambda_{-}\right) \\
1 & g_{+}
\end{array}\right) \\
\underline{e}=e+\lambda_{-}
\end{array}\right.\right.
\end{aligned}
$$

Two zweibeins and two worldsheet coordinates allow two independent worldsheets, so $L_{1}$ is sum of the left and the right sectors. It will be convenient to calculate the $\kappa$-symmetry invariance as shown in the next section.

The Lagrangian $L_{1}$ is rewritten in such a way that the kinetic term becomes 2Ddimensional covariant,

$$
\begin{aligned}
L_{2}= & \frac{1}{e}\left(e_{+}{ }^{m} \partial_{m} X^{M}\right)\left(e_{-}{ }^{n} \partial_{n} X^{N}\right) \hat{\eta}_{M N} \\
& +\left(\frac{1}{\bar{e}}-\frac{1}{e}\right)\left(e_{-}{ }^{m} \partial_{m} X^{\bar{M}}\right)^{2}+\left(\frac{1}{\underline{e}}-\frac{1}{e}\right)\left(e_{+}{ }^{m} \partial_{m} X \underline{M}\right)^{2}
\end{aligned}
$$

where the zweibein $e_{a}{ }^{m}$ is the same one in (3.1). In the conformal gauge $g_{ \pm}=1 L_{2}$ becomes

$$
L_{2}=\frac{1}{2}\left\{\left(\partial_{+} X^{M}\right)\left(\partial_{-} X^{N}\right) \hat{\eta}_{M N}-\frac{\lambda_{+}}{2+\lambda_{+}}\left(\partial_{-} X^{\bar{M}}\right)^{2}-\frac{\lambda_{-}}{2+\lambda_{-}}\left(\partial_{+} X^{\underline{M}}\right)^{2}\right\}
$$


with $\partial_{ \pm}=\partial_{\tau} \pm \partial_{\sigma}$. The selfdual constraints obtained by varying $\lambda_{ \pm}$in terms of $x^{\mathrm{M}}, y_{\mathrm{M}}$ in (2.17) are squares of the usual selfduality condition $\partial_{m} y_{\mathrm{M}}=\epsilon_{m n} \partial^{n} x^{\mathrm{M}}$ equivalently $\partial_{m}(X \eta)^{M}=\epsilon_{m n} \partial^{n} X^{M}$ as

$$
\left\{\begin{array}{l}
\left(\partial_{-} X^{\bar{M}}\right)^{2}=\frac{1}{4}\left(\left(\partial_{\tau} x^{\mathrm{M}}-\partial_{\sigma} y_{\mathrm{M}}\right)+\left(\partial_{\tau} y_{\mathrm{M}}-\partial_{\sigma} x^{\mathrm{M}}\right)\right)^{2}=0 \\
\left(\partial_{+} X^{\underline{M}}\right)^{2}=\frac{1}{4}\left(\left(\partial_{\tau} x^{\mathrm{M}}-\partial_{\sigma} y_{\mathrm{M}}\right)-\left(\partial_{\tau} y_{\mathrm{M}}-\partial_{\sigma} x^{\mathrm{M}}\right)\right)^{2}=0
\end{array}\right.
$$

The D-dimensional dual coordinate $d y_{\mathrm{M}}$ is solved in terms of $d x^{\mathrm{M}}$, if the selfduality constraint is solved. But squares of the selfduality constraints are weaker, and it contributes to make the worldsheet covariance manifest. The Lagrangian $L_{2}$ is rewritten in terms of $x^{\mathrm{M}}, y_{\mathrm{M}}$ in $(2.17)$ as

$$
\begin{aligned}
L_{2}=\frac{1}{e}\left\{\left(e_{+}{ }^{m} \partial_{m} x^{\mathrm{M}}\right)\left(e_{-}{ }^{n} \partial_{n} x^{\mathrm{N}}\right) \eta_{\mathrm{MN}}+\left(e_{+}{ }^{m} \partial_{m} y_{\mathrm{M}}\right)\left(e_{-}{ }^{n} \partial_{n} y_{\mathrm{N}}\right) \eta^{\mathrm{MN}}\right. \\
\left.+\frac{\lambda_{+}}{e+\lambda_{+}}\left(\partial_{\tau} x^{\mathrm{M}}-\partial_{\sigma} y_{\mathrm{M}}\right)\left(\partial_{\sigma} x^{\mathrm{M}}-\partial_{\tau} y_{\mathrm{M}}\right)\right\}
\end{aligned}
$$

in the gauge $\frac{1}{\bar{e}}+\frac{1}{\underline{e}}=\frac{2}{e}$ which is $\lambda_{-}=\frac{e \lambda_{+}}{e+2 \lambda_{+}}$.

The Lagrangian $L_{1}$ is further rewritten in such a way that both the kinetic term and the constraints become 2D-dimensional covariant:

$$
\begin{aligned}
& L_{3}=\frac{1}{\hat{e}}\left(\hat{e}_{+}{ }^{m} \partial_{m} X^{M}\right)\left(\hat{e}_{-}{ }^{n} \partial_{n} X^{N}\right) \hat{\eta}_{M N}+\frac{1}{\hat{\lambda}}\left(\hat{\lambda}_{+}{ }^{m} \partial_{m} X^{M}\right)\left(\hat{\lambda}_{-}{ }^{n} \partial_{n} X^{N}\right) \eta_{M N} \\
& \left\{\begin{array}{l}
\hat{e}_{a}{ }^{m}=\left(\begin{array}{cc}
1 & -\hat{g}_{-} \\
1 & \hat{g}_{+}
\end{array}\right) \\
\hat{g}_{+}=g_{+}+\frac{\lambda_{+} \underline{e}}{\bar{e}+\underline{e}} \\
\hat{g}_{-}=g_{-}+\frac{\lambda_{-} \bar{e}}{\bar{e}+\underline{e}} \\
\hat{e}=2\left(\frac{1}{\bar{e}}+\frac{1}{\underline{e}}\right)^{-1}
\end{array},\left\{\begin{array}{l}
\hat{\lambda}_{a}^{m}=\left(\begin{array}{c}
1-\hat{\lambda}_{-} \\
1 \\
\hat{\lambda}_{+}
\end{array}\right) \\
\hat{\lambda}_{+}=g_{+}+\frac{(2 \bar{e}-e) \underline{e}}{-\bar{e}+\underline{e}} \\
\hat{\lambda}_{-}=g_{-}+\frac{e \bar{e}}{-\bar{e}+\underline{e}} \\
\hat{\lambda}=2\left(\frac{1}{\bar{e}}-\frac{1}{\underline{e}}\right)^{-1}
\end{array}\right.\right.
\end{aligned}
$$

The conformal gauge in the first term is given by

$$
\left\{\begin{array}{l}
\lambda_{+}=e\left(\frac{1}{g_{+}}-1\right) \\
\lambda_{-}=e\left(\frac{1}{g_{-}}-1\right)
\end{array} \rightarrow \hat{e}_{a}^{m}=\left(\begin{array}{cc}
1 & -1 \\
1 & 1
\end{array}\right) .\right.
$$

In this gauge the second term does not allow the conformal gauge, $\hat{\lambda}_{ \pm}=1$, since they are originally selfduality constraints,

$$
\left\{\begin{array} { l } 
{ \hat { \lambda } _ { + } = \frac { 2 ( g _ { + } + g _ { - } - g _ { + } g _ { - } ) } { g _ { + } - g _ { - } } } \\
{ \hat { \lambda } _ { - } = \frac { 2 g _ { + } g _ { - } } { g _ { + } - g _ { - } } }
\end{array} \leftrightarrow \left\{\begin{array}{l}
g_{+}=\frac{2 \hat{\lambda}_{-}}{\hat{\lambda}_{+}+\hat{\lambda}_{-}-2} \\
g_{-}=\frac{2 \hat{\lambda}_{-}}{\hat{\lambda}_{+}+\hat{\lambda}_{-}+2}
\end{array}\right.\right.
$$


This is in contrast to $L_{2}$ in (3.5) where the conformal gauge in the zweibein $e_{a}{ }^{m}$ is allowed for both the kinetic term and the selfduality constraints. The solutions of the selfduality constraints and the section condition lead to the same physical degrees of freedom. The second term should be imposed as the section condition which is the 2D-dimensional covariant orthogonal condition,

$$
\left(\hat{\lambda}_{+}{ }^{m} \partial_{m} X^{M}\right)\left(\hat{\lambda}_{-}^{n} \partial_{n} X^{N}\right) \eta_{M N}=0 .
$$

A solution of the section condition is given in (2.17).

\section{Non-abelian space currents}

\subsection{Algebra and currents}

A nondegenerate graded Lie algebra generated by $G_{I}$ has the nondegenerate group metric $\eta_{I J}$ and the totally graded antisymmetric structure constant $f_{I J K}$

$$
\left[G_{I}, G_{J}\right\}=i f_{I J}{ }^{K} G_{K}, \operatorname{tr}\left(G_{I} G_{J}\right)=\eta_{I J}=\frac{1}{2} \eta_{(I J]}, f_{I J K} \equiv f_{I J}{ }^{L} \eta_{L K}=\frac{1}{3 !} f_{[I J K)}
$$

with the graded bracket $[A, B\}=A B-(-)^{A B} B A$ and the graded symmetrized and antisymmetrized indices $(A, B]=A B+(-)^{A B} B A$ and $[A, B)=A B-(-)^{A B} B A$. A group element $g(Z)$ with coordinates $Z^{I}$ gives two kinds of currents and derivatives: the leftinvariant current $J^{I}$, the particle covariant derivative $\nabla_{I}$, the right-invariant current $\tilde{J}^{I}$ and the particle symmetry generator $\tilde{\nabla}_{I}$ are given by

$$
\begin{aligned}
& \text { Left-invariants : } \quad g^{-1} d g=i J^{I} G_{I}=i d Z^{M} R_{M} G_{I}, \nabla_{I}=\left(R^{-1}\right)_{I}{ }^{M} \frac{1}{i} \frac{\partial}{\partial Z^{M}} \\
& \text { Right-invariants : } d g g^{-1}=i \tilde{J}^{I} G_{I}=i d Z^{M} L_{M} G_{I}, \widetilde{\nabla}_{I}=\left(L^{-1}\right)_{I}^{M} \frac{1}{i} \frac{\partial}{\partial Z^{M}}
\end{aligned}
$$

They satisfy the following Maurer-Cartan equations and the algebras

$$
\begin{array}{lrl}
\text { Left-invariants : } \quad d J^{I}=\frac{1}{2} J^{J} \wedge J^{K} f_{K J}{ }^{I}, & {\left[\nabla_{I}, \nabla_{J}\right\}=-i f_{I J}{ }^{K} \nabla_{K}} \\
\text { Right-invariants : } d \tilde{J}^{I}=-\frac{1}{2} \tilde{J}^{J} \wedge \tilde{J}^{K} f_{K J} J^{I},\left[\widetilde{\nabla}_{I}, \widetilde{\nabla}_{J}\right\} & =i f_{I J}{ }^{K} \widetilde{\nabla}_{K} \\
\text { Mixed } & {\left[\nabla_{I}, \widetilde{\nabla}_{J}\right\}} & =0
\end{array}
$$

The left- and the right-invariant currents are related by the orthogonal matrix $M_{I}{ }^{J}$ as $[9]$

$$
\tilde{J}^{I}=J^{J}\left(M^{-1}\right)_{J}^{I}, \widetilde{\nabla}_{I}=M_{I}^{J} \nabla_{J}, M_{I}^{J}=\left(L^{-1}\right)_{I}{ }^{M} R_{M}{ }^{J}, \eta_{I J}=M_{I}{ }^{K} M_{J}{ }^{L} \eta_{K L} .
$$

The affine extension of the algebras (4.3) is given by generalization $\nabla_{I} \rightarrow \triangleright_{I}(\sigma)$ and $\widetilde{\nabla}_{I} \rightarrow \widetilde{\nabla}_{I}(\sigma)$. The worldsheet indices of the currents are denoted as $m=(\tau, \sigma)=(0,1)$ for currents $J_{m}{ }^{I}$ and $\tilde{J}_{m}{ }^{I}$ with $d Z^{M}=d \sigma^{m} \partial_{m} Z^{M}$. The affine covariant derivative and the symmetry generator are given by

$\begin{gathered}\text { Covariant derivative } \\ (\text { Selfdual current) }\end{gathered} \triangleright_{I}=\nabla_{I}+J_{1}^{J} N_{I}{ }^{K} \eta_{K J}=\nabla_{I}+J_{1}^{J}\left(B_{J I}+\eta_{J I}\right)$

Symmetry generator : $\widetilde{\triangleright}_{I}=\widetilde{\nabla}_{I}-\tilde{J}_{1}^{J} M_{J}^{L} M_{I}^{K} N_{L K}=M_{I}^{J} \hat{\triangleright}_{J}$

Anti-selfdual current $\hat{\triangleright}_{J} \equiv \nabla_{J}+J_{1}^{K}\left(B_{K J}-\eta_{K J}\right)$ 
We consider cases where $B_{I J}$ is constant and determined by the dilatation operator as [9]

$$
B_{I J}=\frac{1}{2} N_{[I \mid}^{K} \eta_{K \mid J)}=\frac{1}{2}\left(n_{J}-n_{I}\right) \eta_{I J}
$$

where the dilatation operator $\hat{N}$ gives the canonical dimension of the generator $G_{I}$ with the following normalization

$$
\left[\hat{N}, G_{I}\right]=i N_{I}^{J} G_{J}=i n_{I} G_{I}, \quad\left(n_{I}+n_{J}\right) \eta_{I J}=2 \eta_{I J}
$$

They satisfy the following affine Lie algebra

$$
\left\{\begin{array}{l}
{\left[\triangleright_{I}(1), \triangleright_{J}(2)\right\}=-i f_{I J}{ }^{K} \triangleright_{K} \delta(2-1)-2 i \eta_{I J} \partial_{\sigma} \delta(2-1)} \\
{\left[\widetilde{\triangleright}_{I}(1), \widetilde{\triangleright}_{J}(2)\right\}=i f_{I J} \widetilde{\triangleright}_{K} \delta(2-1)+2 i \eta_{I J} \partial_{\sigma} \delta(2-1)} \\
{\left[\triangleright_{I}(1), \widetilde{\triangleright}_{J}(2)\right\}=0}
\end{array}\right.
$$

where the worldsheet $\sigma$ coordinates $\sigma_{1}, \sigma_{2}$ are denoted as 1,2 and $\partial_{\sigma} \delta(2-1)=\frac{\partial}{\partial \sigma_{2}} \delta\left(\sigma_{2}-\sigma_{1}\right)$.

In general the Hamiltonian is written as bilinears of the covariant derivatives $\triangleright_{I}$, while the global symmetry charge is given by the integral of the symmetry generator $\tilde{\nabla}_{I}$ so that the Hamiltonian is invariant under the global symmetry. The 2-dimensional operators $\triangleright_{I}$ and $\hat{\nabla}_{I}$, which is $\mathrm{O}(\mathrm{D}, \mathrm{D})$ transformed $\tilde{\nabla}_{I}$ in $(4.5)$, in manifestly T-duality formulation are seldfual and anti-selfdual respectively. Some of the symmetry generators are set to be zero as the dimensional reduction constraints.

\subsection{Virasoro and selfduality constraints}

We double the algebra (4.1) as the direct product of two copies of the algebra with the opposite sign of the structure constant:

$$
\begin{aligned}
G_{I} & \rightarrow G_{I}=\left(G_{\bar{I}}, G_{\underline{I}}\right) \\
Z^{M} & \rightarrow Z^{M}=\left(Z^{\bar{M}}, Z^{\underline{M}}\right) \\
f_{I J}{ }^{K} & \rightarrow f_{I J}{ }^{K}=\left(f_{\bar{I} \bar{J}} \bar{K}, f_{\underline{I J}} \underline{\underline{K}}=-f_{\bar{I} \bar{J}}{ }^{\bar{K}}\right) \\
\eta_{I J} & \rightarrow \eta_{I J}=\left(\eta_{\bar{I} \bar{J}}, \eta_{\underline{I J}}=-\eta_{\bar{I} \bar{J}}\right), \hat{\eta}_{I J}=\left(\eta_{\bar{I} \bar{J}}, \eta_{\underline{I J}}=\eta_{\bar{I} \bar{J}}\right)
\end{aligned}
$$

where two kinds of metrics are written in matrix notarion as

$$
\eta_{I J}=\left(\begin{array}{cc}
\eta_{\bar{I} \bar{J}} & 0 \\
0 & -\eta_{\bar{I} \bar{J}}
\end{array}\right), \hat{\eta}_{I J}=\left(\begin{array}{cc}
\eta_{\bar{I} \bar{J}} & 0 \\
0 & \eta_{\bar{I} \bar{J}}
\end{array}\right)
$$


The bilinears of currents (4.5) contracted with these metrics are the Virasoro generators

$$
\left\{\begin{aligned}
h_{\sigma} & =\frac{1}{4} \triangleright_{I} \eta^{I J} \triangleright_{J}=\frac{1}{4}\left(\left(\triangleright_{\bar{I}}\right)^{2}-\left(\triangleright_{\underline{I}}\right)^{2}\right) \\
h_{\tau} & =\frac{1}{4} \triangleright_{I} \hat{\eta}^{I J} \triangleright_{J}=\frac{1}{4}\left(\left(\triangleright_{\bar{I}}\right)^{2}+\left(\triangleright_{\underline{I}}\right)^{2}\right) \\
\tilde{h}_{\sigma} & =\frac{1}{4} \widetilde{\triangleright}_{I} \eta^{I J} \widetilde{\triangleright}_{J}=\frac{1}{4}\left(\left(\widetilde{\triangleright}_{\bar{I}}\right)^{2}-\left(\widetilde{\triangleright}_{\underline{I}}\right)^{2}\right) \\
& =\frac{1}{4} \hat{\triangleright}_{I} \eta^{I J} \hat{\triangleright}_{J}=\frac{1}{4}\left(\left(\hat{\triangleright}_{\bar{I}}\right)^{2}-\left(\hat{\triangleright}_{\underline{I}}\right)^{2}\right) \\
\tilde{h}_{\tau} & =\frac{1}{4} \widetilde{\triangleright}_{I} \hat{\eta}^{I J} \widetilde{\triangleright}_{J}=\frac{1}{4}\left(\left(\widetilde{\triangleright}_{\bar{I}}\right)^{2}+\left(\widetilde{\triangleright}_{\underline{I}}\right)^{2}\right)
\end{aligned}\right.
$$

where $\widetilde{\triangleright}_{I}=M_{I}^{J} \hat{\triangleright}_{J}$ in (4.5) is used in the fourth line. The Virasoro algebras are given by

$$
\begin{aligned}
& \left\{\begin{array}{l}
{\left[h_{\sigma}(1), h_{\sigma}(2)\right]=-i\left(h_{\sigma}(1)+h_{\sigma}(2)\right) \partial_{\sigma} \delta(2-1)} \\
{\left[h_{\sigma}(1), h_{\tau}(2)\right]=-i\left(h_{\tau}(1)+h_{\tau}(2)\right) \partial_{\sigma} \delta(2-1)} \\
{\left[h_{\tau}(1), h_{\tau}(2)\right]=-i\left(h_{\sigma}(1)+h_{\sigma}(2)\right) \partial_{\sigma} \delta(2-1)}
\end{array}\right. \\
& \left\{\begin{array}{l}
{\left[\tilde{h}_{\sigma}(1), \tilde{h}_{\sigma}(2)\right]=i\left(\tilde{h}_{\sigma}(1)+\tilde{h}_{\sigma}(2)\right) \partial_{\sigma} \delta(2-1)} \\
{\left[\tilde{h}_{\sigma}(1), \tilde{h}_{\tau}(2)\right]=i\left(\tilde{h}_{\tau}(1)+\tilde{h}_{\tau}(2)\right) \partial_{\sigma} \delta(2-1)} \\
{\left[\tilde{h}_{\tau}(1), \tilde{h}_{\tau}(2)\right]=i\left(\tilde{h}_{\sigma}(1)+\tilde{h}_{\sigma}(2)\right) \partial_{\sigma} \delta(2-1)}
\end{array}\right. \\
& \begin{cases}{\left[h_{m}(1), \tilde{h}_{n}(2)\right]=0, \quad m, n=(\tau, \sigma)}\end{cases}
\end{aligned}
$$

Derivative operators act on fields $\Phi(Z)$ as $Z^{M}$ derivatives

$$
\left[\triangleright_{I}, \Phi\right]=\left[\hat{\triangleright}_{I}, \Phi\right]=\frac{1}{i}\left(R^{-1}\right)_{I}^{M} \partial_{M} \Phi(Z)
$$

The $\sigma$ derivative is defined by the commutator with both selfdual and anti-selfdual Virasoro operators, so that the $\sigma$ derivative in canonical formalism coincides with the usual chain rule derivative

$$
\partial_{\sigma} \Phi(Z)=i\left[\int\left(h_{\sigma}-\tilde{h}_{\sigma}\right), \Phi\right]=\frac{1}{2}\left(\triangleright_{I}-\hat{\triangleright}_{I}\right) \eta^{I J}\left(R^{-1}\right)_{I}^{M} \partial_{M} \Phi=\partial_{\sigma} Z^{M} \partial_{M} \Phi
$$

Therefore we take a set of constraints for a string system in the doubled space; the Virasoro constraints and the selfduality constraints as

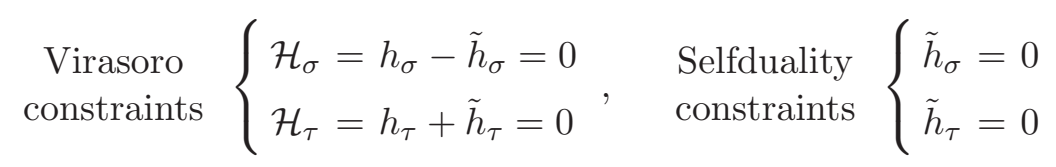

$\mathcal{H}_{\sigma}$ and $\mathcal{H}_{\tau}$ satisfy the same Virasoro algebra in (4.12). 


\section{$5 \quad$ T-dual superstring Lagrangians}

\subsection{Superalgebras and currents}

In the manifestly T-duality formulation a superstring in a flat space is governed by the doubled nondegenerate superalgebra generated by $G_{\mathcal{M}}$. The doubled indices for the left and right sectors are denoted by $\mathcal{M}=(\overline{\mathcal{M}}, \underline{\mathcal{M}})$. The nondegenerate supergenerators are denoted as $G_{\mathcal{M}}=\left(d_{\mu}, P_{M}, \omega^{\mu}\right)=\left(d_{\bar{\mu}}, P_{\bar{M}}, \omega^{\bar{\mu}} ; d_{\underline{\mu}}, P_{\underline{M}}, \omega^{\underline{\mu}}\right)$. The algebra is given by

$$
\begin{aligned}
\text { Left : } \quad\left\{d_{\bar{\mu}}, d_{\bar{\nu}}\right\}=2 P_{\bar{M}} \gamma^{\bar{M}}{ }_{\bar{\mu} \bar{\nu}}, \quad\left[d_{\bar{\mu}}, P_{\bar{M}}\right]=2\left(\gamma_{\bar{M}} \omega\right)_{\bar{\mu}} \\
\text { Right }:\left\{d_{\underline{\mu}}, d_{\underline{\nu}}\right\}=-2 P_{\underline{M}} \gamma_{\underline{M}}^{\underline{\mu} \underline{\mu}}, \quad\left[d_{\underline{\mu}}, P_{\underline{M}}\right]=-2\left(\gamma_{\underline{M}} \omega\right)_{\underline{\mu}}
\end{aligned}
$$

The nondegenerate metric $\eta_{M N}$ and $B_{M N}$ are given as

$$
\begin{aligned}
& \eta_{\mathcal{M N}}=\left(\begin{array}{c|c}
\eta_{\overline{\mathcal{M N}}} & 0 \\
\hline 0 & -\eta_{\overline{\mathcal{M N}}}
\end{array}\right), \quad \quad \eta_{\overline{\mathcal{M N}}}=\begin{array}{c}
d_{\bar{\mu}} \\
P_{\bar{M}} \\
\omega^{\bar{\mu}}
\end{array}\left(\begin{array}{r}
1 \\
1
\end{array}\right) \\
& B_{\mathcal{M N}}=\left(\begin{array}{c|c}
B_{\overline{\mathcal{M N}}} & 0 \\
\hline 0 & -B_{\overline{\mathcal{M N}}}
\end{array}\right), \quad \quad B_{\overline{\mathcal{M N}}}=\begin{array}{c}
d_{\bar{\mu}} \\
P_{\bar{M}} \\
\omega^{\bar{\mu}}
\end{array}\left(\begin{array}{c}
{ }^{\frac{1}{2}} \\
\frac{1}{2}
\end{array}\right)
\end{aligned}
$$

where blank spaces are zeros.

For a group element $g$ the left-invariant and the right-invariant currents are denoted by

$$
\begin{aligned}
& \frac{1}{i} g^{-1} d g=J^{\mathcal{M}} G_{\mathcal{M}}=J^{\bar{\mu}} d_{\bar{\mu}}+J^{\bar{M}} P_{\bar{M}}+J_{\bar{\mu}} \omega^{\bar{\mu}}+J^{\underline{\mu}} d_{\underline{\mu}}+J \underline{M} P_{\underline{M}}+J_{\underline{\mu}} \omega^{\underline{\mu}} \\
& \frac{1}{i} d g g^{-1}=\tilde{J}^{\mathcal{M}} G_{\mathcal{M}}=\tilde{J}^{\bar{\mu}} d_{\bar{\mu}}+\tilde{J}^{\bar{M}} P_{\bar{M}}+\tilde{J}_{\bar{\mu}} \omega^{\bar{\mu}}+\tilde{J}^{\mu} d_{\underline{\mu}}+\tilde{J}^{\underline{M}} P_{\underline{M}}+\tilde{J}_{\underline{\mu}} \omega^{\underline{\mu}}
\end{aligned}
$$

The Maurer-Cartan equations for the left/right one form currents are given as

Left-invariant currents

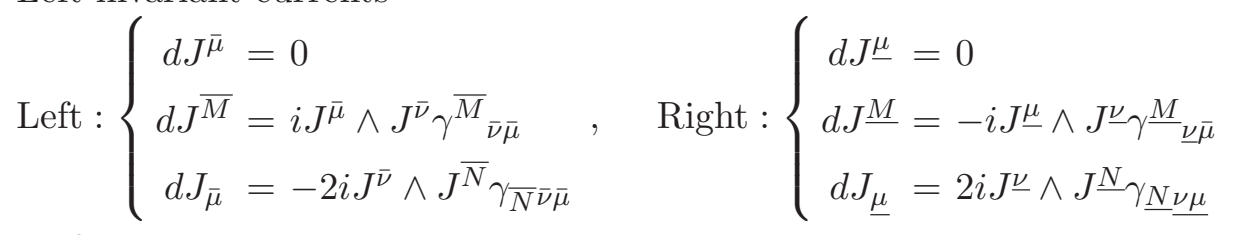

Right-invariant currents

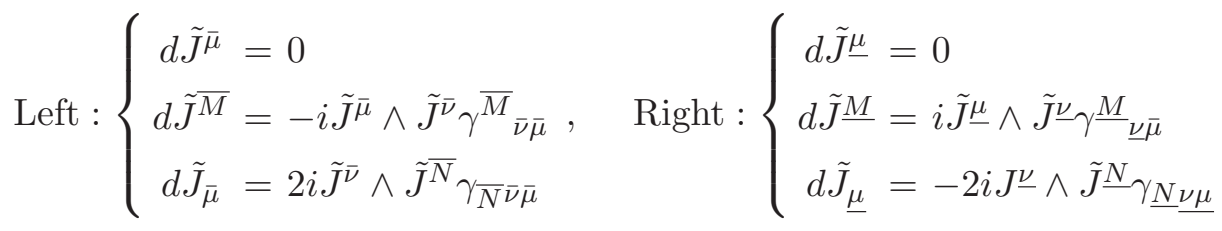

A group element $g\left(Z^{\mathcal{M}}\right)$ is parametrized with $Z^{\mathcal{M}}=\left(\theta^{\bar{\mu}}, X^{\bar{M}}, \varphi_{\bar{\mu}} ; \theta^{\underline{\mu}}, X^{\underline{X}}, \varphi_{\underline{\mu}}\right)$ by

$$
\begin{aligned}
& g\left(Z^{\mathcal{M}}\right)=g\left(Z^{\overline{\mathcal{M}}}\right) g\left(Z^{\underline{\mathcal{M}}}\right) \\
& \left\{\begin{array}{l}
g\left(Z^{\overline{\mathcal{M}}}\right)=\exp \left(i \varphi_{\bar{\mu}} \omega^{\bar{\mu}}\right) \exp \left(i X^{\bar{M}} P_{\bar{M}}\right) \exp \left(i \theta^{\bar{\mu}} d_{\bar{\mu}}\right) \\
g(Z \underline{\mathcal{M}})=\exp \left(i \varphi_{\underline{\mu}} \omega^{\underline{\mu}}\right) \exp \left(i X^{\underline{M}} P_{\underline{M}}\right) \exp \left(i \theta^{\underline{\mu}} d_{\underline{\mu}}\right)
\end{array}\right.
\end{aligned}
$$


The left/right-invariant currents are given as

Left-invariant currents

$$
\begin{aligned}
& \text { Left : }\left\{\begin{array}{l}
J^{\bar{\mu}}=d \theta^{\bar{\mu}} \\
J^{\bar{M}}=d X^{\bar{M}}-i \theta \gamma^{\bar{M}} d \theta \\
J_{\bar{\mu}}=d \varphi_{\bar{\mu}}-2 i\left(d X^{\bar{M}}-\frac{i}{3} \theta \gamma^{\bar{M}} d \theta\right)\left(\theta \gamma_{\bar{M}}\right)_{\bar{\mu}}
\end{array}\right. \\
& \text { Right : }\left\{\begin{array}{l}
J^{\underline{\mu}}=d \theta^{\underline{\mu}} \\
J^{\underline{M}}=d X \underline{M}+i \theta \gamma^{\underline{M}} d \theta \\
J_{\underline{\mu}}=d \varphi_{\underline{\mu}}+2 i\left(d X \underline{\underline{M}}+\frac{i}{3} \theta \gamma^{\underline{M}} d \theta\right)\left(\theta \gamma_{\underline{M}}\right)_{\underline{\mu}}
\end{array}\right.
\end{aligned}
$$

Right-invariant currents

$$
\begin{aligned}
& \text { Left : }\left\{\begin{array}{l}
\tilde{J}^{\bar{\mu}}=d \theta^{\bar{\mu}} \\
\tilde{J}^{\bar{M}}=d X^{\bar{M}}+i\left(\theta \gamma^{\bar{M}} d \theta\right) \\
\tilde{J}_{\bar{\mu}}=d \varphi_{\bar{\mu}}-2 i\left(d X^{\bar{M}}-\frac{i}{3} \theta \gamma^{\bar{M}} d \theta\right)\left(\theta \gamma_{\bar{M}}\right)_{\bar{\mu}}
\end{array}\right. \\
& \text { Right : }\left\{\begin{array}{l}
\tilde{J}^{\underline{\mu}}=d \theta^{\underline{\mu}} \\
\tilde{J}^{\underline{M}}=d X^{\underline{M}}+i \theta \gamma^{\underline{M}} d \theta \\
\tilde{J}_{\underline{\mu}}=d \varphi_{\underline{\mu}}+2 i\left(d X \underline{\underline{M}}+\frac{i}{3} \theta \gamma^{\underline{M}} d \theta\right)\left(\theta \gamma_{\underline{M}}\right)_{\underline{\mu}}
\end{array}\right.
\end{aligned}
$$

The left/right-invariant derivatives are given as

Left-invariant currents

$$
\begin{aligned}
& \text { Left : }\left\{\begin{array}{l}
\nabla_{\bar{\mu}}=-i \partial_{\bar{\mu}}+\left(\theta \gamma^{\bar{M}}\right)_{\bar{\mu}} \partial_{\bar{M}}+i \frac{4}{3}\left(\theta \gamma^{\bar{M}}\right)_{\bar{\mu}}\left(\theta \gamma_{\bar{M}}\right)_{\bar{\nu}} \partial^{\bar{\nu}} \\
\nabla_{\bar{M}}=-i \partial_{\bar{M}}+2\left(\theta \gamma^{\bar{M}}\right)_{\bar{\mu}} \partial^{\bar{\nu}} \\
\nabla^{\bar{\mu}}=-i \partial^{\bar{\mu}}
\end{array}\right. \\
& \text { Right : }\left\{\begin{array}{l}
\nabla_{\underline{\mu}}=-i \partial_{\underline{\mu}}-\left(\theta \gamma^{\underline{M}}\right)_{\underline{\mu}} \partial_{\underline{M}}+i \frac{4}{3}\left(\theta \gamma^{\underline{M}}\right)_{\underline{\mu}}\left(\theta \gamma_{\underline{M}}\right)_{\underline{\nu}} \partial^{\underline{\nu}} \\
\nabla_{\underline{M}}=-i \partial_{\underline{M}}-2\left(\theta \gamma^{\underline{M}}\right)_{\underline{\nu}} \partial^{\underline{\nu}} \\
\nabla_{\underline{\mu}}=-i \partial^{\underline{\mu}}
\end{array}\right.
\end{aligned}
$$

Right-invariant currents

$$
\begin{aligned}
& \text { Left : }\left\{\begin{array}{l}
\widetilde{\nabla}_{\bar{\mu}}=-i \partial_{\bar{\mu}}-\left(\theta \gamma^{\bar{M}}\right)_{\bar{\mu}} \partial_{\bar{M}}+2 X_{\bar{M}} \gamma^{\bar{M}}{ }_{\bar{\mu} \bar{\nu}} \partial^{\bar{\nu}}-i \frac{2}{3}\left(\theta \gamma^{\bar{M}}\right)_{\bar{\mu}}\left(\theta \gamma_{\bar{M}}\right)_{\bar{\nu}} \partial^{\bar{\nu}} \\
\widetilde{\nabla}_{\bar{M}}=-i \partial_{\bar{M}} \\
\widetilde{\nabla}^{\bar{\mu}}=-i \partial^{\bar{\mu}}
\end{array}\right. \\
& \text { Right : }\left\{\begin{array}{l}
\widetilde{\nabla}_{\underline{\mu}}=-i \partial_{\underline{\mu}}+(\theta \gamma \underline{M})_{\underline{\mu}} \partial_{\underline{M}}-2 X_{\underline{M}} \gamma^{\underline{M}} \underline{\mu} \underline{\underline{\nu}} \partial^{\underline{\nu}}-i \frac{2}{3}\left(\theta \gamma^{\underline{M}}\right)_{\underline{\mu}}\left(\theta \gamma_{\underline{M}}\right)_{\underline{\nu}} \partial^{\underline{\nu}} \\
\widetilde{\nabla}_{\underline{M}}=-i \partial_{\underline{M}} \\
\widetilde{\nabla}_{\underline{\mu}}^{\mu}=-i \partial^{\underline{\mu}}
\end{array}\right.
\end{aligned}
$$

The covariant derivatives $\nabla_{\mathcal{M}}$ and the anti-selfdual currents $\hat{\nabla}_{\mathcal{M}}$ which are proportional 
to the symmetry generators $\widetilde{\nabla}_{\mathcal{M}}=M_{\mathcal{M}} \mathcal{N}^{\hat{D}_{\mathcal{N}}}$ as in (4.3) and (4.4) are the followings:

Selfdual current, $\nabla_{\mathcal{M}}=\left(D_{\mu}, \mathcal{P}_{M}, \Omega^{\mu}\right)$

Left $:\left\{\begin{array}{l}D_{\bar{\mu}}=\nabla_{\bar{\mu}}-\frac{1}{2} J_{1 \bar{\mu}} \\ \mathcal{P}_{\bar{M}}=\nabla_{\bar{M}}+J_{1 \bar{M}} \\ \Omega^{\bar{\mu}}=\nabla^{\bar{\mu}}+\frac{3}{2} J_{1}^{\bar{\mu}}\end{array}\right.$, Right $:\left\{\begin{array}{l}D_{\underline{\mu}}=\nabla_{\underline{\mu}}+\frac{1}{2} J_{1 \underline{\mu}} \\ \mathcal{P}_{\underline{M}}=\nabla_{\underline{M}}-J_{1 \underline{M}} \\ \Omega_{\underline{\mu}}=\nabla \underline{\mu}-\frac{3}{2} J_{1} \underline{\mu}\end{array}\right.$

Anti-selfdual currents, $\hat{\nabla}_{\mathcal{M}}=\left(\hat{D}_{\mu}, \hat{\mathcal{P}}_{M}, \hat{\Omega}^{\mu}\right)$

Left $:\left\{\begin{array}{l}\hat{D}_{\bar{\mu}}=\nabla_{\bar{\mu}}+\frac{3}{2} J_{1 \bar{\mu}} \\ \hat{\mathcal{P}}_{\bar{M}}=\nabla_{\bar{M}}-J_{1 \bar{M}} \\ \hat{\Omega}^{\bar{\mu}}=\nabla^{\bar{\mu}}-\frac{1}{2} J_{1} \bar{\mu}\end{array}\right.$, Right $:\left\{\begin{array}{l}\hat{\delta}_{\underline{\mu}}=\nabla_{\underline{\mu}}-\frac{3}{2} J_{1 \underline{\mu}} \\ \hat{\mathcal{P}}_{\underline{M}}=\nabla_{\underline{M}}+J_{1 \underline{M}} \\ \hat{\Omega}^{\underline{\mu}}=\nabla^{\underline{\mu}}+\frac{1}{2} J_{1} \underline{\mu}\end{array}\right.$

\subsection{Superstring Lagrangians}

We propose the superstring Lagrangian with manifestly T-duality in Hamilton form

$$
\begin{aligned}
& L=\partial_{\tau} Z^{\mathcal{M}} P_{\mathcal{M}}-H
\end{aligned}
$$

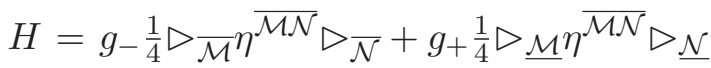

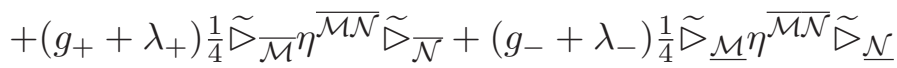

$$
\begin{aligned}
& +\bar{\Lambda} \widetilde{\Omega}^{\bar{\mu}}+\underline{\Lambda} \widetilde{\Omega}^{\mu}
\end{aligned}
$$

where the set of first class constraints are the Virasoro constraints, the selfduality constraints and the dimensional reduction constraints of auxiliary fermions

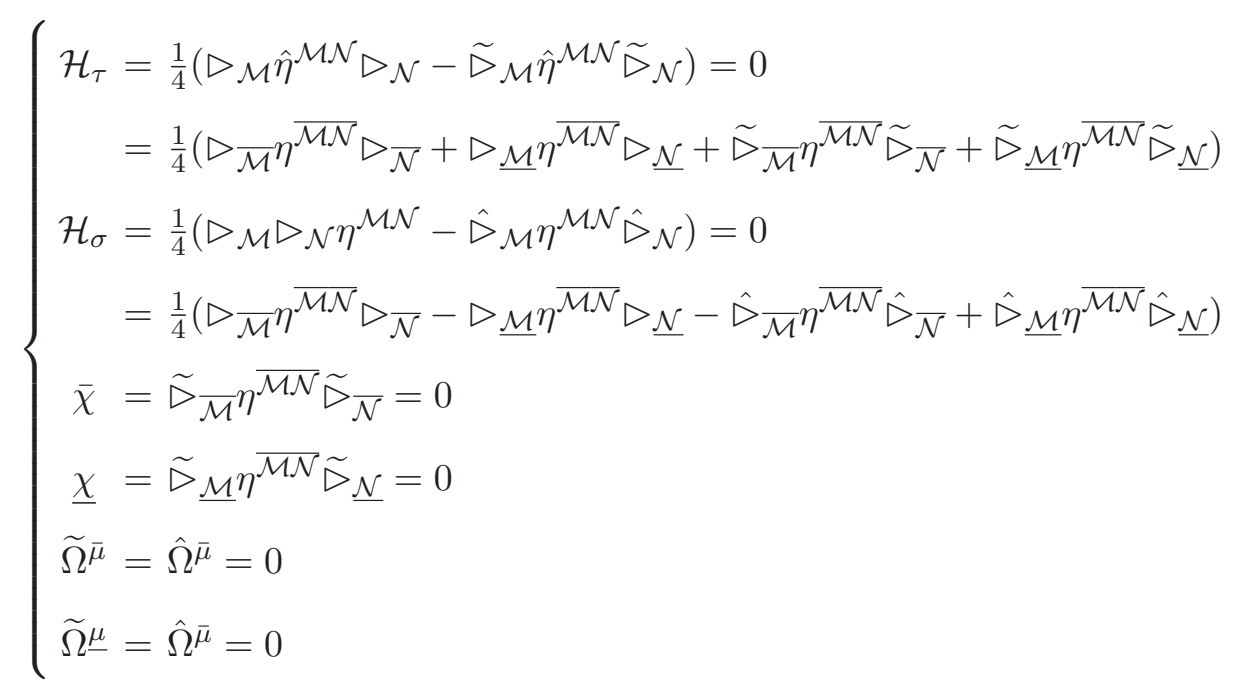

In the last two lines $M^{\mu \nu}=0=M^{\mu}{ }_{N}$ and $M^{\mu}{ }_{\nu}=\delta_{\mu}^{\nu}$ of $M_{\mathcal{M}^{\mathcal{N}}}=\left(L^{-1} R\right)_{\mathcal{M}^{\mathcal{N}}}$ are used. The Hamiltonian becomes

$$
\begin{aligned}
H= & g_{-}\left(\frac{1}{4} \mathcal{P}_{\bar{M}}{ }^{2}+\frac{1}{2} \Omega^{\bar{\mu}} D_{\bar{\mu}}\right)+g_{+}\left(\frac{1}{4} \mathcal{P}_{\underline{\underline{M}}}^{2}+\frac{1}{2} \Omega^{\underline{\mu}} D_{\underline{\mu}}\right)+\left(g_{+}+\lambda_{+}\right) \frac{1}{4} \hat{\mathcal{P}}_{\bar{M}}{ }^{2}+\left(g_{-}+\lambda_{-}\right) \frac{1}{4} \hat{\mathcal{P}}_{\underline{M}^{M}}{ }^{2} \\
& +\bar{\Lambda} \widetilde{\Omega}^{\bar{\mu}}+\underline{\Lambda} \widetilde{\Omega}^{\underline{\mu}}
\end{aligned}
$$


The Legendre tranfsormation brings to the following Lagrangian

$$
\begin{aligned}
& L_{1}=L_{1: \mathrm{kin}}+L_{1: \mathrm{WZ}} \\
& \left\{\begin{aligned}
L_{1: \mathrm{kin}} & =\frac{1}{\overline{\bar{e}}}\left(\bar{e}_{+}{ }^{m} J_{m}^{\bar{M}}\right)\left(\bar{e}_{-}{ }^{n} J_{n}^{\bar{N}}\right) \eta_{\overline{M N}}+\frac{1}{\underline{e}}\left(\underline{e}_{+}{ }^{m} J_{m} \underline{M}\right)\left(\underline{e}_{-}{ }^{n} J_{n} \underline{N}\right) \eta_{\overline{M N}} \\
L_{1: \mathrm{WZ}} & =\frac{k}{2}\left(J_{[0}{ }^{\bar{\mu}} J_{1] \bar{\mu}}-J_{[0} \underline{\underline{\mu}}_{1] \underline{\mu}}\right) \\
& =\frac{k}{2}\left[\underline{\bar{e}}^{\bar{e}}\left(\bar{e}_{[+\mid}{ }^{m} J_{m}^{\bar{\mu}}\right)\left(\bar{e}_{\mid-]^{n}} J_{n \bar{\mu}}\right)-\frac{\underline{1}}{\underline{e}}\left(\underline{e}_{[+\mid}{ }^{m} J_{m} \underline{\mu}\right)\left(\underline{e}_{\mid-]^{n}} J_{n \underline{\mu}}\right)\right]
\end{aligned}\right.
\end{aligned}
$$

This is one of the main results, the superstring Lagrangian with manifest T-duality with double zweibeins where $\bar{e}_{a}{ }^{m}$ and $\underline{e}_{a}{ }^{m}$ are zweibeins in (3.4). The worldsheet contraction $\epsilon_{a b}=\frac{1}{e} e_{a}{ }^{m} e_{b}{ }^{n} \epsilon_{m n}=\frac{1}{\bar{e}} \bar{e}_{a}{ }^{m} \bar{e}_{b}{ }^{n} \epsilon_{m n}=\frac{1}{e} \underline{e}_{a}{ }^{m} \underline{e}_{b}{ }^{n} \epsilon_{m n}$ with $\epsilon_{01}=-\epsilon_{10}=1$ is used. The Hamiltonian (5.17) gives $k=1$, and we generalize the constant $k= \pm 1$ as usual.

Doubling zweibein makes computation of the $\kappa$-symmetry transformation easier, since each left and right sector Lagrangians are $\kappa$-symmetry invariant separately. The $\kappa$ symmetry transformation $\delta_{\kappa} g$ is denoted by $\Delta^{\mathcal{M}}$ and the vector components $\Delta^{M}$ vanish as

$$
g^{-1} \delta_{\kappa} g=i \Delta^{\mathcal{M}} G_{\mathcal{M}}, \quad \Delta^{M}=\left(\Delta^{\bar{M}}, \Delta^{\underline{M}}\right)=0 .
$$

Since the left and right sectors are completely independent, we just use $e_{a}{ }^{m}$ as the zweibeins instead of $\bar{e}_{a}{ }^{m}$ and $\underline{e}_{a}{ }^{m}$ to perform the $\kappa$-invariance computation only in this paragraph. The $\kappa$-symmetry transformation of zwiebein $e_{m}{ }^{a}$ is denoted as

$$
\delta_{\kappa}\left(\frac{1}{\sqrt{e}} e_{a}^{m}\right) \sqrt{e} e_{m}^{b}=\Delta_{a}^{b}
$$

which is traceless, $\Delta_{a}{ }^{a}=0$. The worldsheet currents with the zwiebein is denoted as $J_{a}{ }^{\mathcal{M}}=\frac{1}{\sqrt{e}} e_{a}{ }^{m} J_{m}{ }^{\mathcal{M}}$. Under the $\kappa$-symmetry transformation the currents are transformed as

$$
\delta_{\kappa} J_{m}{ }^{\mathcal{M}}=\partial_{m} \Delta^{\mathcal{M}}+J_{m}{ }^{\mathcal{L}} \Delta^{\mathcal{N}} f_{\mathcal{N} \mathcal{L}}{ }^{\mathcal{M}}, \quad \delta_{\kappa} J_{a}{ }^{\mathcal{M}}=\Delta_{a}{ }^{b} J_{b}{ }^{\mathcal{M}}+\partial_{a} \Delta^{\mathcal{M}}+J_{a}{ }^{\mathcal{L}} \Delta^{\mathcal{N}} f_{\mathcal{N} \mathcal{L}^{\mathcal{M}}}
$$

with $\partial_{a}=\frac{1}{\sqrt{e}} e_{a}{ }^{m} \partial_{m}$. It is convenient to write down the $\kappa$-symmetry transformation of the left and right currents

$$
\begin{aligned}
& \text { Left : } \quad\left\{\begin{aligned}
\delta_{\kappa} J_{a}^{\bar{\mu}}=\Delta_{a}{ }^{b} J_{b} \bar{\mu}+\partial_{a} \Delta^{\bar{\mu}} \\
\delta_{\kappa} J_{a}^{\bar{M}}=\Delta_{a}^{b} J_{b} \bar{M}+2 i \Delta^{\bar{\mu}} J_{a}{ }^{\bar{\nu}} \gamma^{\bar{M}}{ }_{\bar{\mu} \bar{\nu}} \\
\delta_{\kappa} J_{a \bar{\mu}}=\Delta_{a}{ }^{b} J_{b \bar{\mu}}+\partial_{a} \Delta_{\bar{\mu}}-2 i \Delta^{\bar{\nu}} J_{a}{ }^{\bar{M}} \gamma_{\bar{M} \bar{\mu} \bar{\nu}}
\end{aligned}\right. \\
& \text { Right : }\left\{\begin{array}{l}
\delta_{\kappa} J_{a} \underline{\underline{\mu}}=\Delta_{a}^{b} J_{b} \underline{\underline{\mu}}+\partial_{a} \Delta^{\underline{\mu}} \\
\delta_{\kappa} J_{a} \underline{\underline{M}}=\Delta_{a}^{b} J_{b} \underline{\underline{M}}-2 i \Delta^{\underline{\mu}} J_{a}^{\underline{\underline{\nu}}} \gamma^{\underline{\underline{M}}} \underline{\underline{\mu} \underline{\underline{\nu}}} \\
\delta_{\kappa} J_{a \bar{\mu}}=\Delta_{a}^{b} J_{b \underline{\mu}}+\partial_{a} \Delta_{\underline{\mu}}+2 i \Delta^{\underline{\underline{\nu}}} J_{a}^{\underline{M}} \gamma_{\underline{M} \underline{\mu} \underline{\underline{\mu}}}
\end{array}\right.
\end{aligned}
$$

Under the $\kappa$-symmetry transformation the left sector Lagrangian, with $\bar{J}$ and $\bar{\Delta}$ corresponding to $\bar{e}_{a}{ }^{m}$, becomes

$$
\left.\delta_{\kappa} L\right|_{\text {Left }}=\bar{\Delta}_{++}\left(\overline{J_{-}^{\bar{M}}}\right)^{2}+\bar{\Delta}_{--}\left(\bar{J}_{+}^{\bar{M}}\right)^{2}+2 i \bar{\Delta}^{\bar{\mu}} \gamma_{\bar{M} \bar{\mu} \bar{\nu}}\left((1+k) \bar{J}_{+}^{\bar{\nu}} \bar{J}_{-}^{\bar{M}}+(1-k) \bar{J}_{-}^{\bar{\nu}} \bar{J}_{+}^{\bar{M}}\right)
$$


up to total derivative terms. The Maurer-Cartan equation in (5.5) was used. Let us set the $\kappa$-symmetry parameter for the left sector $\bar{\kappa}$ as

$$
\bar{\Delta}^{\bar{\mu}}=\frac{1}{2}(1+k) \bar{J}_{-}^{\bar{M}} \gamma_{\bar{M}} \bar{\mu}^{\bar{\mu}} \bar{\kappa}_{+; \bar{\nu}}+\frac{1}{2}(1-k) \bar{J}_{+}^{\bar{M}} \gamma_{\bar{M}}{ }^{\bar{\mu}} \bar{\nu}_{-; \bar{\nu}}
$$

Then it becomes

$$
\left.\delta_{\kappa} L\right|_{\text {Left }}=\left(\bar{\Delta}_{++}+2 i(1+k) \bar{\kappa}_{+; \bar{\mu}} \bar{J}_{+}{ }^{\bar{\mu}}\right)\left(\bar{J}_{-}^{\bar{M}}\right)^{2}+\left(\bar{\Delta}_{--}+2 i(1-k) \bar{\kappa}_{-; \bar{\mu}} \bar{J}_{-} \bar{\mu}^{\bar{\mu}}\right)\left(\bar{J}_{+}^{\bar{M}}\right)^{2}
$$

Therefore the $\kappa$-symmetry invariance requires the transformation of the zweibein as

$$
0=\bar{\Delta}_{++}+2 i(1+k) \bar{\kappa}_{+; \bar{\mu}} \bar{J}_{+}{ }^{\bar{\mu}}=\bar{\Delta}_{--}+2 i(1-k) \bar{\kappa}_{-; \bar{\mu}} \bar{J}_{-}{ }^{\bar{\mu}} .
$$

Analogously the $\kappa$-symmetry transformations for the right sector with $\underline{J}, \underline{\Delta}$ and $\underline{\kappa}$ are given as

$$
0=\underline{\Delta}_{++}-2 i(1-k) \underline{\kappa}_{+; \underline{\mu}} \underline{J}_{+}{ }^{\underline{\mu}}=\underline{\Delta}_{--}-2 i(1+k) \underline{\kappa}_{-; \underline{\mu}} \underline{J}_{-} \underline{\underline{\mu}} .
$$

The Lagrangian $L_{1}$ is rewritten in a $2 \mathrm{D}$-dimensional covariant way as $L_{2}$ in (3.5). Then the manifestly T-duality covariant superstring Lagrangian is given by

$$
\begin{aligned}
& L_{2}=L_{2: \mathrm{kin}}+L_{2: \mathrm{SD}}+L_{2: \mathrm{WZ}} \\
& \left\{\begin{array}{l}
L_{2: \mathrm{kin}}=\frac{1}{e}\left(e_{+}{ }^{m} J_{m}^{M}\right)\left(e_{-}{ }^{n} J_{n}^{N}\right) \hat{\eta}_{M N} \\
L_{2: \mathrm{SD}}=\left(\frac{1}{\bar{e}}-\frac{1}{e}\right)\left(e_{-}{ }^{n} J_{n}^{\bar{M}}\right)^{2}+\left(\frac{1}{\underline{e}}-\frac{1}{e}\right)\left(e_{+}{ }^{n} J_{\bar{n}}\right)^{2} \\
\left.L_{2: \mathrm{WZ}}=\frac{k}{2}\left(J_{[0}{ }^{\bar{\mu}} J_{1] \bar{\mu}}\right)-J_{[0} \underline{\underline{\mu}} J_{1] \underline{\mu}}\right)
\end{array}\right.
\end{aligned}
$$

The kinetic term has both the 2D-target space covariance and the worldsheet covariance.

Next let us examine how the Lagrangian reduces into the Green-Schwarz superstring Lagrangian. The left and right Lorentz symmetries can be fixed in such a way that two gamma matrices are identified to the D-dimensional gamma matrix as $\gamma^{\bar{M}}{ }_{\bar{\mu}}=-\gamma \underline{\underline{M}}_{\mu \nu}=$ $\gamma_{\mu \nu}^{\mathrm{M}}$. The left and right spinors $\theta^{\bar{\mu}}$ and $\theta \underline{\underline{\mu}}$ can be chosen to be the opposite chirality for the type IIA and the same chirality for the type IIB theories. In terms of $x^{\mathrm{M}}, y_{\mathrm{M}}$ coordinates in (2.17), the currents are written as

$$
\left\{\begin{array}{l}
\mathbf{J}_{m}{ }^{\mathrm{M}}=J_{m}^{\bar{M}}+J_{m}^{\underline{M}}=\partial_{m} x^{\mathrm{M}}-i\left(\theta^{\bar{\mu}} \gamma^{\mathrm{M}}{ }_{\mu \nu} \partial_{m} \theta^{\bar{\nu}}+\theta \underline{\mu}^{\mathrm{M}}{ }_{\mu \nu} \partial_{m} \theta^{\underline{\nu}}\right) \\
\mathbf{J}_{m \mathrm{M}}=J_{m}^{\bar{M}}-J_{m} \underline{\underline{M}}=\partial_{m} y_{\mathrm{M}}-i\left(\theta^{\bar{\mu}} \gamma_{\mathrm{M} \mu \nu} \partial_{m} \theta^{\bar{\nu}}-\theta^{\underline{\mu}} \gamma_{\mathrm{M} \mu \nu} \partial_{m} \theta^{\underline{\nu}}\right) \\
J_{m}{ }^{\bar{\mu}}=\partial_{m} \theta^{\bar{\mu}} \\
J_{m} \underline{\mu}=\partial_{m} \theta^{\underline{\mu}} \\
J_{m ; \bar{\mu}}=\partial_{m} \varphi_{\bar{\mu}}-i\left(2 \partial_{m}(x+y)^{\mathrm{M}}-\frac{2 i}{3} \theta^{\bar{\nu}} \gamma^{\mathrm{M}}{ }_{\bar{\nu} \bar{\rho}} \partial_{m} \theta^{\bar{\rho}}\right)\left(\theta \gamma_{\mathrm{M}}\right)_{\bar{\mu}} \\
J_{m ; \underline{\mu}}=\partial_{m} \varphi_{\underline{\mu}}-i\left(2 \partial_{m}(x-y)^{\mathrm{M}}-\frac{2 i}{3} \theta_{\underline{\nu}} \gamma_{\underline{\nu \rho}}^{\mathrm{M}} \partial_{m} \theta \underline{\rho}\right)\left(\theta \gamma_{\mathrm{M}}\right)_{\underline{\mu}}
\end{array}\right.
$$


Then the Lagrangian $L_{2}$ is rewritten as

$$
\begin{aligned}
& L_{2}=L_{2: \mathrm{kin}}+L_{2: \mathrm{SD}}+L_{2: \mathrm{WZ}} \\
& \left\{\begin{aligned}
L_{2: \mathrm{kin}}= & \frac{1}{2 e}\left\{\left(e_{+}{ }^{m} \mathbf{J}_{m}{ }^{\mathrm{M}}\right)\left(e_{-}{ }^{n} \mathbf{J}_{n}{ }^{\mathrm{N}}\right) \eta_{\mathrm{MN}}+\left(e_{+}{ }^{m} \mathbf{J}_{m \mathrm{M}}\right)\left(e_{-}{ }^{n} \mathbf{J}_{n \mathrm{~N}}\right) \eta^{\mathrm{MN}}\right\} \\
L_{2: \mathrm{SD}}= & \frac{\lambda}{4 e}\left\{\left(e_{+}{ }^{m}+e_{-}{ }^{m}\right) \mathbf{J}_{m}{ }^{\mathrm{M}}-\left(e_{+}{ }^{m}-e_{-}{ }^{m}\right) \mathbf{J}_{m \mathrm{M}}\right\} \\
& \times\left\{-\left(e_{+}{ }^{m}-e_{-}{ }^{m}\right) \mathbf{J}_{m}{ }^{\mathrm{M}}+\left(e_{+}{ }^{m}+e_{-}{ }^{m}\right) \mathbf{J}_{m \mathrm{M}}\right\} \\
L_{2: \mathrm{WZ}}= & \left.\frac{k}{2}\left(J_{[0}{ }^{\bar{\mu}} J_{1] \bar{\mu}}\right)-J_{\left[0^{\underline{\mu}}\right.} J_{1] \underline{\mu}}\right)
\end{aligned}\right.
\end{aligned}
$$

with $\lambda=\frac{\lambda_{+}}{e+\lambda_{+}}$. The selfduality constraint $L_{2 ; \mathrm{SD}}$ in the conformal gauge $g_{ \pm}=1$ is given by

$$
L_{2: \mathrm{SD}}=\frac{\lambda}{2}\left(\mathbf{J}_{0}^{\mathrm{M}}-\mathbf{J}_{1 \mathrm{M}}\right)\left(\mathbf{J}_{1}^{\mathrm{M}}-\mathbf{J}_{0 \mathrm{M}}\right)
$$

where the selfduality condition for a superstring is

$$
\mathbf{J}_{m}{ }^{N} \hat{\eta}_{N M}=\epsilon_{m n} \mathbf{J}_{n}{ }^{N} \eta_{N M}
$$

The zweibein squared is the worldsheet metric $g^{m n}=e_{a}{ }^{m} e_{b}^{n} \rho^{a b}$ with $-\rho^{00}=1=\rho^{11}$ and $g=\operatorname{det} g_{m n}$. Then kinetic term $L_{2: \text { kin }}$ is given as

$$
L_{2: \text { kin }}=-\frac{1}{2} \sqrt{g}\left\{g^{m n} \mathbf{J}_{m}{ }^{\mathrm{M}} \mathbf{J}_{n}{ }^{\mathrm{N}} \eta_{\mathrm{MN}}+g^{m n} \mathbf{J}_{m \mathrm{M}} \mathbf{J}_{n \mathrm{~N}} \eta^{\mathrm{MN}}\right\}
$$

The Wess-Zumino term is given as

$$
\begin{aligned}
L_{2: \mathrm{WZ}}=\frac{k}{2} \epsilon^{m n}\{ & \left(\partial_{m} \theta^{\bar{\mu}} \partial_{n} \varphi_{\bar{\mu}}-\partial_{m} \theta^{\mu} \partial_{n} \varphi_{\underline{\mu}}\right) \\
& \left.-\partial_{m} x^{\mathrm{M}} i\left(\theta^{\bar{\mu}} \partial_{n} \theta^{\bar{\nu}}-\theta^{\underline{\mu}} \partial_{n} \theta^{\underline{\nu}}\right) \gamma_{\mathrm{M} \mu \nu}-\partial_{m} y_{\mathrm{M}} i\left(\theta^{\bar{\mu}} \partial_{n} \theta^{\bar{\nu}}+\theta^{\underline{\mu}} \partial_{n} \theta^{\underline{\nu}}\right) \gamma_{\mathrm{M} \mu \nu}\right\}
\end{aligned}
$$

The Green-Schwarz superstring Lagrangian is obtained from (5.31) by the gauge $\lambda=0$ and a section with $\mathbf{J}_{m \mathrm{M}}=0$;

$$
\begin{aligned}
& L_{2}=L_{2: \mathrm{kin}}+L_{2: \mathrm{WZ}} \\
& \left\{\begin{array}{l}
L_{2: \mathrm{kin}}=-\frac{1}{2} \sqrt{g} g^{m n} \mathbf{J}_{m}{ }^{\mathrm{M}} \mathbf{J}_{n}{ }^{\mathrm{N}} \eta_{\mathrm{MN}} \\
L_{2: \mathrm{WZ}}=\frac{k}{2} \epsilon^{m n}\left\{\left(\partial_{m} \theta^{\bar{\mu}} \partial_{n} \varphi_{\bar{\mu}}-\partial_{m} \theta^{\underline{\mu}} \partial_{n} \varphi_{\underline{\mu}}\right)\right. \\
\left.\quad-i \partial_{m} x^{\mathrm{M}}\left(\theta^{\bar{\mu}} \partial_{n} \theta^{\bar{\nu}}-\theta^{\mu} \partial_{n} \theta^{\underline{\nu}}\right) \gamma_{\mathrm{M} \mu \nu}+\left(\theta^{\bar{\mu}} \gamma^{\mathrm{M}}{ }_{\mu \nu} \partial_{m} \theta^{\bar{\nu}}\right)\left(\theta^{\underline{\rho}} \gamma_{\mathrm{M} \rho \lambda} \partial_{n} \theta^{\underline{\lambda}}\right)\right\}
\end{array}\right.
\end{aligned}
$$

The auxiliary fermions $\varphi_{\mu}$ 's appear only in the surface terms which are absent in the usual Green-Schwarz superstring action, so $\varphi_{\mu}$ 's are gauged away. It is interesting that the bosonic Wess-Zumino term $\epsilon^{m n} \mathbf{J}_{m}{ }^{\mathrm{M}} \mathbf{J}_{n \mathrm{M}}$ does not show up contrasting to the chiral approach [9]. 


\section{Conclusions}

In this paper we have presented Lagrangians with manifest T-duality for the type II superstring. The chiral scalar problem is solved by adding the anti-selfdual currents. While only the selfdual currents are physical, the unphysical anti-selfdual currents are also included for the worldsheet covariance of both the Weyl and Lorentz symmetries. The selfduality constraints are imposed to suppress the degrees of freedom of the anti-selfdual currents whose Lagrange multipliers become the worldsheet zweibeins. Then the Lagrangian has double zweibeins and so double worldsheets. Double zweibeins in the superstring Lagraigian make the type II $\kappa$-symmetry splitting into two sets of the type I $\kappa$-symmetries leading to simpler computation of $\kappa$-symmetry. Resulted superstring Lagrangian with manifest T-duality including double zweibeins is (5.18), the one with single zweibein is (5.29) and the one in terms of the usual $x^{\mathrm{M}}, y_{\mathrm{M}}$ coordinates with single zweibein is (5.31). The superstring Lagrangians are simple structure; all terms are given in bilinears of symmetry invariant currents manifesting the global supersymmetry, the T-duality symmetry, coordinate invariance and the $\kappa$-symmetry. We give the gauge condition and the section condition which lead to the Green-Schwarz superstring Lagrangian.

Including the Ramond-Ramond field based on the central extended superalgebra [21] and constructing D-brane Lagrangians are future problems. Superstrings and D-branes with background fields will be also interesting.

\section{Acknowledgments}

M.H. would like to thank the Simons Center for Geometry and Physics for hospitality during "the 2019 Summer Simons workshop in Mathematics and Physics" where this work has been developed. W.S. is supported by NSF grant PHY-1620628.

\section{A Indices}

Indices are summarized.

$$
\begin{aligned}
& \text { Integer }: \text { Nonabelian space } \cdots I, J, \cdots \\
& \text { Caligraphy }: \text { Superspace } \cdots \mathcal{M}, \mathcal{N}, \cdots \\
& \text { Middle }: \text { doubled vector } \cdots M, N, \cdots \\
& \text { greek }: \text { doubled spinors } \cdots \mu, \nu, \cdots \\
& \text { UPPER CASE }: \text { spacetime } \cdots M, N, \cdots \\
& \text { lower case }: \text { worldvolume } \cdots m, n, \cdots \\
& \bar{B} \text { arred }: \text { left-handed } \cdots \bar{M}, \bar{N}, \cdots \\
& \underline{\text { Underlined }}: \text { right-handed } \cdots \underline{M}, \underline{N}, \cdots \\
& \text { Roman }: \text { D-dimensional vector } \cdots \mathrm{M}, \mathrm{N}, \cdots
\end{aligned}
$$

Open Access. This article is distributed under the terms of the Creative Commons Attribution License (CC-BY 4.0), which permits any use, distribution and reproduction in any medium, provided the original author(s) and source are credited. 


\section{References}

[1] M.J. Duff, Duality rotations in string theory, Nucl. Phys. B 335 (1990) 610 [INSPIRE].

[2] A.A. Tseytlin, Duality symmetric formulation of string world sheet dynamics, Phys. Lett. B 242 (1990) 163 [INSPIRE].

[3] A.A. Tseytlin, Duality symmetric closed string theory and interacting chiral scalars, Nucl. Phys. B 350 (1991) 395 [InSPIRE].

[4] P. Pasti, D.P. Sorokin and M. Tonin, Duality symmetric actions with manifest space-time symmetries, Phys. Rev. D 52 (1995) R4277 [hep-th/9506109] [INSPIRE].

[5] P. Pasti, D.P. Sorokin and M. Tonin, On Lorentz invariant actions for chiral $p$ forms, Phys. Rev. D 55 (1997) 6292 [hep-th/9611100] [InSPIRE].

[6] D.S. Berman, N.B. Copland and D.C. Thompson, Background field equations for the duality symmetric string, Nucl. Phys. B 791 (2008) 175 [arXiv:0708.2267] [INSPIRE].

[7] I. Bandos, Superstring in doubled superspace, Phys. Lett. B 751 (2015) 408 [arXiv:1507.07779] [INSPIRE].

[8] M. Hatsuda and W. Siegel, $O(D, D)$ gauge fields in the T-dual string Lagrangian, JHEP 02 (2019) 010 [arXiv: 1810.04761] [INSPIRE].

[9] M. Hatsuda, K. Kamimura and W. Siegel, Type II chiral affine Lie algebras and string actions in doubled space, JHEP 09 (2015) 113 [arXiv:1507.03061] [INSPIRE].

[10] M. Hatsuda, K. Kamimura and W. Siegel, Superspace with manifest T-duality from type-II superstring, JHEP 06 (2014) 039 [arXiv:1403.3887] [INSPIRE].

[11] C.D.A. Blair, E. Malek and A.J. Routh, An $O(D, D)$ invariant Hamiltonian action for the superstring, Class. Quant. Grav. 31 (2014) 205011 [arXiv:1308.4829] [INSPIRE].

[12] J.-H. Park, Green-Schwarz superstring on doubled-yet-gauged spacetime, JHEP 11 (2016) 005 [arXiv: 1609.04265] [INSPIRE].

[13] J.-I. Sakamoto and Y. Sakatani, Local $\beta$-deformations and Yang-Baxter $\sigma$-model, JHEP 06 (2018) 147 [arXiv: 1803.05903] [INSPIRE].

[14] R. Borsato and L. Wulff, Non-Abelian T-duality and Yang-Baxter deformations of Green-Schwarz strings, JHEP 08 (2018) 027 [arXiv: 1806.04083] [INSPIRE].

[15] B. Nikolić and B. Sazdović, Advantage of the second-order formalism in double space T-dualization of type-II superstring, Eur. Phys. J. C 79 (2019) 819 [arXiv:1907.03571] [INSPIRE].

[16] W. Siegel, Manifest duality in low-energy superstrings, in International Conference on Strings '93, Berkeley, CA, U.S.A., 24-29 May 1993, pg. 353 [hep-th/9308133] [INSPIRE].

[17] W. Siegel, Superspace duality in low-energy superstrings, Phys. Rev. D 48 (1993) 2826 [hep-th/9305073] [INSPIRE].

[18] W. Siegel, Two vierbein formalism for string inspired axionic gravity, Phys. Rev. D 47 (1993) 5453 [hep-th/9302036] [INSPIRE].

[19] O. Hohm, W. Siegel and B. Zwiebach, Doubled $\alpha^{\prime}$-geometry, JHEP 02 (2014) 065 [arXiv: 1306.2970] [INSPIRE].

[20] A. Sen, Self-dual forms: action, Hamiltonian and compactification, J. Phys. A 53 (2020) 084002 [arXiv:1903.12196] [InSPIRE].

[21] M. Hatsuda, K. Kamimura and W. Siegel, Ramond-Ramond gauge fields in superspace with manifest T-duality, JHEP 02 (2015) 134 [arXiv: 1411.2206] [INSPIRE]. 